\title{
FEASIBILITY ANALYSIS OF DOUBLE-HIT HAMMERING FOR PILE FOUNDATIONS
}

\author{
Michel Cotsaftis ${ }^{1}$, Erno Keskinen ${ }^{2}$, Sirpa Launis ${ }^{2}$, Yrjo \\ Raunisto $^{3}$ \\ ${ }^{1}$-Ecole Centrale d'Electronique, Laboratoire des Techniques Mecatroniques et \\ Electroniques 53 rue de Grenelle 75007 Paris France, email : mcot@ece.fr \\ 2 -Tampere University of Technology, Laboratory of Machine Dynamics PO Box \\ 589, FIN-33101 Tampere, Finland, email : erno.keskinen@tut.fi \\ ${ }^{3}$-Unisto Oy, Tolkkimaentie 9, FIN-13130 Hameenlinna, Finland, email : \\ unisto@kolumbus.fi
}

\begin{abstract}
The problem of the realization of a double hit hammer for inserting piles in the ground as needed in construction has been addressed. It is mainly shown that in parameter space of the mechanical system, there exists an operating window where the single cycle behavior corresponding to one stroke of the power system can be repeated in a stable periodic behavior with period equal to the single cycle one. As the applied control is a simple one, it would be of interest to consider technical experiments to check the usefulness of the concept.
\end{abstract}

\section{I - Introduction}

Construction and technical modern realizations are requiring to pile always larger elements in the ground, and to deal with more difficult problems related to size and weight of manipulated objects to pile. Development of a gripper system has allowed the hammering force to be acting very close to the ground and to reduce pile deformations and vibrations. Efficiency is increased by this method, but the fundamental question of improving the overall system itself remains open, as classically the penetration depth is relatively modest for the power injected and the hammer mass involved. Improvement from using vibratory drivers[1] is limited, as, though limiting noise level, the power input at each stroke is reduced so that it rapidly stays below a threshold value increasing with the size of the piled object. In a different way, it has been suggested[2] to split hammer mass into two mechanically interrelated masses, so that at each stroke the hit on hammer is itself splitted into two hits acting on each mass. It is then interesting to determine whether a carefully designed mechanical link between the two masses could produce a significant change in hammering efficiency with this type of structure, and to verify later whether such system is technically workable within the limits of today technology. This in particular implies to make sure that the cycle is repeatable a large enough number of times so that the double hit hammering can be processed for a complete piling run. The repeatability rests upon the fact that during the second part of the elementary cycle after their collision, the two masses are effectively moving together up to the final stopping time, and that the next stroke on exterior mass is itself coming at the right time so that during the next following cycle, the collision time is not shifted away from the optimum single cycle value. Mathematically, these conditions are expressing the periodicity of the complete run over a cycle. From their analytical representation, the restriction of existence domain of the three system parameters can be discussed to define the workable window.

\section{II - System Equations}

Instead of a classical one piece hammer of mass $M$, consider a two-component one with exterior hollow component of mass $M_{1}$ moved by hydraulic servo, and an interior one of mass $M_{2}$ moving with low friction inside the cavity of first component and mechanically linked to it. Then one should determine the command which enhances at most the efficiency of such system, being understood that there are two aspects in first, the static piling effect, and in second, the realization of the dynamical sequence allowing to repeat for many cycles the elementary double hammering stroke. A passive case will be considered here where the link is be represented by a spring of stiffness $K$, see Fig.1. From system definition, dynamical equations for each component are of generic form 


$$
\begin{aligned}
& M_{1} \frac{d^{2} x_{1}}{d t^{2}}+F_{1}\left(\frac{d x_{1}}{d t}, x_{1}, x_{2}\right)+R=0 \\
& M_{2} \frac{d^{2} x_{2}}{d t^{2}}+F_{2}\left(\frac{d x_{2}}{d t}, x_{2}, x_{1}\right)=0
\end{aligned}
$$

with $x_{1}, x_{2}$ the positions of each component, $R$ the resistance to penetration of the ground, and $F_{l}, F_{2}$ the forces mainly due to friction affecting components dynamics coming from their interaction and from piled object-soil interaction[3].

Owing to the small velocities during motion, these forces will be linearized in their arguments in (1) as

$$
\begin{aligned}
& F_{1}=f_{1} \frac{d x_{1}}{d t}+\alpha K x_{1}+K\left(x_{1}-x_{2}\right) \\
& F_{2}=f_{2}\left(\frac{d x_{2}}{d t}-\frac{d x_{1}}{d t}\right)+K\left(x_{1}-x_{2}\right)
\end{aligned}
$$

Initial conditions are

$$
\begin{aligned}
& x_{1}(0)=0, M_{1} \frac{d x_{1}}{d t}(0)=P \\
& x_{2}(0)=0, M_{2} \frac{d x_{2}}{d t}(0)=\beta P
\end{aligned}
$$

where hammer hit is assimilable to a percussion at time $t=0$ with resulting momentum $P$, of which a part $\beta P$ is directly communicated through all mechanical links to the second internal component. The system $(1,3)$ can be nondimensionalized with characteristic pene-tration time $\theta=p / r$ and penetration depth $\pi=$ $p^{2} / r$ where $p=P / M_{1}$ and $r=R / M_{l}$ as

$$
\begin{aligned}
& \frac{d^{2} \eta_{1}}{d \tau^{2}}+\phi_{1} \frac{d \eta_{1}}{d \tau}+\alpha \kappa_{1} \eta+\kappa\left(\eta_{1}-\eta_{2}\right)+1=0 \\
& \mu \frac{d^{2} \eta_{2}}{d \tau^{2}}+\phi_{2}\left(\frac{d \eta_{2}}{d \tau}-\frac{d \eta_{1}}{d \tau}\right)+\kappa\left(\eta_{1}-\eta_{2}\right)=0
\end{aligned}
$$

with initital conditions $\eta_{1}(0)=0, \eta^{{ }^{\prime}}{ }_{1}(0)=1, \eta_{2}(0)=$ $0, \eta_{2}^{\prime}(0)=\beta$, and defining $t=\theta \tau, x_{j}=\pi \eta_{j}, k=K / M_{1}$, $\mu=M_{2} / M_{1}, f_{j}=\theta \phi_{j}, k \theta^{2}=\kappa$. After mass collision at convenient time $\tau_{C}$, the two masses will be supposed to stay together $\left(\eta_{1}=\eta_{2}\right)$ - both conditions which will impose restrictions on possible values of system coefficients as seen later -, and obey the equation

$$
(1+\mu) \frac{d^{2} \eta}{d \tau^{2}}+\phi_{1} \frac{d \eta}{d \tau}+\alpha \kappa \eta 1=0
$$

with initial conditions $\eta\left(\tau_{C}\right)=\eta_{1}\left(\tau_{C}\right)=\eta_{1 C}, \eta^{\prime}\left(\tau_{C}\right)=$ $\eta^{\prime}{ }_{1 C}+(1+\mu)^{-1}\left(\eta_{2 C}{ }_{2 C} \eta^{\prime}{ }_{1 C}\right)$. Eqn.(5) should be solved up to the time $\tau_{F}$ for which $\eta^{\prime}\left(\tau_{F}\right)=0$ corresponding to trajectory end point for one elementary cycle.
It should now be verified that this cycle is repeatable, which amounts to reconsider the system $(4,5)$ with the new periodic initial conditions

$$
\begin{array}{ll}
\eta_{1}(0)=0, & \eta_{1}^{\prime}(0)=\sum_{n} \delta(\tau-n T) \\
\eta_{2}(0)=0, & \eta_{2}^{\prime}(0)=\beta \sum_{n} \delta(\tau-n T)
\end{array}
$$

where $T$ is the time interval between two strokes, and to determine the parameter domain for which hit efficiency is larger than with conventional one mass system.

\section{III - Periodic System Analysis}

The success of the two-mass approach thus rests upon satisfaction of different conditions concerning both the elementary one stroke cycle and the continuation over as many cycles as possible. In the first group is the possibility for the second mass component, starting slower than the first one, to take advantage of spring force to hit the cavity bottom end with a relative velocity as large as possible and before the end of first mass component motion, hence the name double hit. This problem has been previously analyzed[4] and one gets in nondissipative case the analytical expressions

$$
\begin{aligned}
& \eta_{F}=\eta\left(\tau_{F}\right)=\eta_{F 0}\left(2 \tau_{C}\left[1+\beta-\tau_{C} / 2\right]+\right. \\
& \left.\left[1-\tau_{C}+2 \beta+\gamma-\mu\right]^{2}\right) \equiv \eta_{F 0} Q(\beta, \mu, \omega) \\
& \tau_{F}=1+\gamma+2 \beta-\mu \\
& \gamma(1+\mu)=\frac{1-\cos \left(\omega \tau_{C}\right)}{\omega \sin \left(\omega \tau_{C}\right)}
\end{aligned}
$$

where for convience $\gamma=1-\beta / \mu$ and $\omega=(\kappa / \mu)^{1 / 2}$, out of which the normalized final one-cycle time $\tau_{F}$ and the normalized one-cycle penetration depth $\eta_{F}$ can be calculated in terms of system parameters $\omega \mu$ and initial condition $\beta$, and compared to their values $\tau_{F 0}=$ 1 and $\eta_{F 0}=1 /(2[1+\mu])$ for the classical one-mass hammer with same total mass and same initial stroke. The two-mass system will be more efficient than the one-mass one if final penetration depth per time is larger after the same time interval, ie if $Q(\beta, \mu, \omega) / \tau_{F}$ $>1$ with larger value. This condition is easily satisfied for large parameter range with typically a value 1.5 for the ratio. Also it should be verified that collision occurs before the first mass reaches its rest position and that after collision the two masses are staying in contact during all the second phase of the motion. In non dissipative case the first condition writes $\tau_{C}<$ $2(1+\mu \beta)$ and is to be combined with (8) to eliminate $\tau_{C}$ . The second condition can be expressed as $\eta_{2}(\tau)>$ $\eta(\tau)$ for $\tau_{C} \leq \tau \leq \tau_{F}$ where $\eta_{2}(\tau)$ is the solution for second mass motion during the first period $\tau<\tau_{C}$ and extended to the next interval. The condition 
reduces to $\eta_{2}\left(\tau_{F}\right)>\eta\left(\tau_{F}\right)$ as a consequence of the dynamical behavior of the two masses. In the non dissipative case a simple sufficient condition takes the form $\omega\left(\tau_{F}-\tau_{C}\right)<\pi$ ie

$$
\omega\left([1+\mu \beta] \tau_{C}-\tau_{C}^{2} / 2\right)<\pi
$$

So when (9) is satisfied with the two conditions

$$
\begin{gathered}
\tau_{C}<2(1+\mu \beta), \\
\operatorname{Max} Q(\beta, \mu, \omega) / \tau_{F}>1
\end{gathered}
$$

the system returns to equilibrium position at time $\tau_{F}$. So for any $\mathrm{T} \geq \tau_{F}$ the system will have a periodic behavior.

More generally, it is useful to consider the case where the two masses rest positions are not coincident and differ by a normalized distance $a$. In this situation, at time $\tau_{\mathrm{F}}$ the second mass is off its equilibrium position and for $\tau>\tau_{\mathrm{F}}$ the system is running again according to the equations

$$
\begin{aligned}
& \eta_{1}+\mu \eta_{2}=\mu \eta_{F}-\frac{1}{2}\left(\tau-\tau_{F}\right)^{2} \\
& \eta_{2}-\eta_{1}=\frac{1}{\omega^{2}}+\left(\eta_{F}-\frac{1}{\omega^{2}}\right) \cos \omega\left(\tau-\tau_{F}\right)
\end{aligned}
$$

and there exists a time $T$ for which $\eta_{1}(T)=\eta_{2}(T)=0$ ie

$$
\begin{aligned}
& \mathrm{T}-\tau_{F}=(2 \mu a)^{1 / 2}, \\
& \cos (2 \mu a)^{1 / 2} \omega=\left(1-a \omega^{2}\right)^{-1}
\end{aligned}
$$

defining $a=\eta_{F}$ and $T$. Note that the velocities at $T$ are not 0 , so if the system receives a stroke at $T$, the initial velocities are now $\eta_{10}^{\prime}=1+\eta_{1}^{\prime}(T), \quad \eta_{20}^{\prime}=\beta / \mu+\eta_{2}^{\prime}(T)$, but the important point is that at collision between the two masses the supplementary stroke due to second mass is given by $\Delta_{C}^{\prime}=\left|\eta_{10}^{\prime}-\eta_{20}^{\prime}\right|$ only depending on initial velocities. So if from eqn $(8) \tau_{C}$ is fixed instead of $\omega$ for convenience of optimization, it can be verified from eqns $(11,12)$ that after the time interval $T$ the system returns to the same state ie there is also a periodic solution to the initial system in this case.

\section{IV - Stability Considerations}

Though there exists a continuous family of periodic solutions to the piecewise linear system $(4,5)$, not all of them are acceptable for double hit optimization purpose. First one should select the solution corresponding to the shortest period ie to minimize the distance between mass rest positions compatible with eqns $(9,10)$ and technical constraints related to mass decoupling. Another requirement is stability to prevent damageable blow out of dynamical behavior due to inadequate timing of hammer strokes. First it can easily be verified that the system $(4,5)$ is locally stable as the coefficients of the characteristic equations

$$
\begin{aligned}
& \mu s^{4}+\left(\phi_{2}+\mu \phi\right) s^{3}+\left[\kappa+(1+\alpha) \mu \quad \kappa+\phi_{1} \phi_{2}\right] s^{2} \\
& +\kappa\left(\phi_{1}+\alpha \quad \phi_{2}\right) s+\alpha \quad \kappa^{2}=0 \\
& (1+\mu) s^{2}+\phi_{1} s+\alpha \quad \kappa=0
\end{aligned}
$$

are such that the conditions for negative real values of their solutions, which reduce to $\alpha \kappa>0$ and to $\mu+[1-$ $\left.(\alpha \mu)^{1 / 2}\right]^{2}>0$, are all satisfied. Two trajectories with close initial points will remain close, but only within a domain of continuity of the equations.

Starting now from nominal initial values corresponding to unperturbed periodic trajectory, one will analyze how close trajectories starting in its vicinity will evolve during one period. This amounts to use Poincaré map[5] of initial ball and verify conditions for which its transforms after a number of periods are

in a bounded enough domain. However this BIBO condition may be too loose and a more restricted contraction condition after one single period will be studied. So considering the nominal sequence

$$
\begin{aligned}
& \left\{\begin{array}{cc}
(0,0) & 0 \\
1+\eta_{1}^{\prime} & \beta / \mu \\
\hline & \eta_{2}^{\prime}
\end{array}\right\} \rightarrow\left\{\begin{array}{ll}
\eta_{c} & \tau_{c} \\
\eta_{c}^{\prime} &
\end{array}\right\} \rightarrow \\
& \left\{\begin{array}{cc}
\left(\eta_{F}, a\right) & \tau_{F} \\
0 &
\end{array}\right\} \rightarrow\left\{\begin{array}{cc}
(0,0) & T \\
1+\eta_{1}^{\prime} & \beta / \mu+\eta_{2}^{\prime}
\end{array}\right\}
\end{aligned}
$$

characterizing the previous periodic trajectory, let us suppose that there is a perturbation $\left(\delta \eta_{j 0}, \delta \eta_{j 0}{ }^{\prime}\right)$ of initial point. After the first time interval one gets at time $\tau_{c}$

$$
\begin{aligned}
& \delta \eta_{c}=\delta \hat{\eta}_{0}+\tau_{c} \delta \hat{\eta}_{0}^{\prime}+\left(\hat{\eta_{0}^{\prime}}-\tau_{c}\right) \delta \tau_{c} \\
& \delta \eta_{c}^{\prime}=\delta \eta_{0}^{\prime}-\delta \tau_{c}
\end{aligned}
$$

with

$$
\delta \tau_{c}=\frac{\delta \bar{\eta}_{0}^{\prime}}{\omega \Delta_{0}^{\prime}} \sin \omega \tau-\frac{\delta \bar{\eta}_{0}}{\Delta_{0}^{\prime}} \cos \omega \varepsilon
$$

and the definitions

$$
\begin{aligned}
& \delta \hat{\eta}_{0}=(1+\mu)^{-1}\left(\delta \eta_{10}+\mu \delta \eta_{0}\right) \\
& \delta \overline{\eta_{0}}=\delta \eta_{20}-\delta \eta_{10}, \Delta_{0}^{\prime}=\eta_{10}^{\prime}-\eta_{20}^{\prime} .
\end{aligned}
$$

During the second part of the motion where the two masses are in contact, the error at collision propagates and at $\tau_{F}$ one gets

$$
\begin{aligned}
& \delta \eta_{F}=\delta \eta_{C}+\eta_{C}^{\prime} \delta \eta_{C}^{\prime}, \\
& \delta \eta_{F}^{\prime}=\delta \eta_{C}^{\prime}+\delta \tau_{C}=\delta \eta_{0}^{\prime}, \\
& \delta \tau_{F}=\delta \hat{\eta}_{C}^{\prime}+\delta \tau_{C}
\end{aligned}
$$


where the errors are calculated at time $\tau_{F}$. For the last part, where the inner mass returns to its initial value located at abscissa $a$, the propagation of the errors gives at the end of the period $T$ the shift

$$
\begin{aligned}
& \delta \eta_{2 T}=a\left(1-\cos \omega \quad \delta_{F^{\tau}}\right) \cong 0 \\
& \delta \eta_{2 T}^{\prime}=a \omega \sin \omega \delta_{F^{\tilde{t}}} \cong a \omega^{2} \delta \tau_{F}
\end{aligned}
$$

The displacement error is 0 to first order. Consequently, the role of the last part of the elementary cycle is to shield inner mass motion against small enough perturbations, and a small initial displacement $\left(\delta \eta_{20}, \delta \eta^{\prime}{ }_{20}\right)$ thus becomes $\left(0, a \omega^{2} \delta \tau_{F}\right)$. For the perturbation to disappear, one should impose a contraction property ie that , $\delta \eta_{20}{ }_{20}>a \omega^{2} \delta \tau_{F}$, which with eqns $(17,18)$ simply writes

$$
a<\frac{1+\mu}{\mu \omega^{2}}
$$

This condition expresses the constraint that the equilibrium distance between the two masses is upper bounded for stability of the two mass system. The system is thus technically viable with fixed period $T$ if this distance is realizable. An order of magnitude for the right hand side of eqn(19) is .lin normalized units, ie a limit value for $a$ is $\left(.1 p^{2} / r\right)$. As there is an inferior technical limit depending on the mass value for the equilibrium distance between the masses, there exists a minimum value of the normalized stroke $p$ below which such a control cannot be used. This is corresponding to small hammering units. For them it is necessary to operate the system in a different way. Instead of having a constant period $T$, one should depart the next stroke each time the masses are at their initial equilibrium positions. Technically it is sufficient to have an electric contact at equilibrium position of the inner mass which initiates the stroke with included delay effect to account for transmission-execution time. As a consequence the period between two consecutive strokes is no longer a fixed constant but varies depending on the shift with respect to nominal value. In all cases, there exists a window in parameter space within which the system exhibits a stable behavior, ie can be operated for a large number of strokes. Numerical calculations of the model are in progress.

\section{V-Conclusion}

The problem of operating a piling hammer in double hit mode, in which the hammer mass is split into two mechanically related masses, one receiving the blow and the other one being moved and colliding the first one with larger velocity to give an extra impulsion, has been previously addressed and was showing interesting performance enhancement over a single stroke operation. Here the repeatability of this operation has been studied, and it has been mainly shown that there exists interesting windows in system parameter space so that the system exhibits stable behavior. Part of these windows, corresponding to a natural periodic system behavior, is resulting from adequate design of nominal mechanical parameters, and is in principle directly accessible in technical terms. Because it may be limited in applications, there is another part aside which does not correspond to a strictly periodic behavior, as it is resulting from the repeatability of the displacement of inner mass and its crossing of equilibrium position. In this case, a control system to check the crossing with adapted delay in the loop is needed. Depending on case study, both ways have their interest to be decided on experimental observations.

\section{References}

1- J.E. Bowles : Foundation Analysis and Design, McGraw-Hill, Singapore, 1988

2- E.K. Keskinen, M. Keskiniva, M. Raitaniemi, Y. Raunisto : Design and Analysis of a Multipurpose Double-acting Hammering Unit for Hydraulic Excavators, Proc. ASME Engineering System Design and Analysis Conference, Montpellier, France, 1996

3- T. Whittaker : The Design of Piled Foundations, Pergamon Press, Oxford, 1976

4- M. Cotsaftis, E.K. Keskinen, S. Launis, Y. Raunisto : Performance Analysis of Double Hit Piler System, Proc. of ETCE/OMAE 2000 Joint Conference, ASME, Feb. 14-17 2000, New-Orleans, LA

5- N. Minorski : Nonlinear Oscillations, D. Van Nostrand, Princeton, N.J., 1962 